Article

\title{
Wind Tunnel Study on Wake Instability of Twin H-Rotor Vertical-Axis Turbines
}

\author{
Kun Wang ${ }^{1}$, Li Zou ${ }^{1,2,3, *}$, Aimin Wang ${ }^{1}{ }^{1}$, Peidong Zhao ${ }^{1}$ and Yichen Jiang ${ }^{1, *}$ \\ 1 School of Naval Architecture, Dalian University of Technology, Dalian 116024, China; \\ kunwang@mail.dlut.edu.cn (K.W.); wam@mail.dlut.edu.cn (A.W.); zhaopeidong@mail.dlut.edu.cn (P.Z.) \\ 2 State Key Laboratory of Structural Analysis for Industrial Equipment, Dalian University of Technology, \\ Dalian 116024, China \\ 3 Collaborative Innovation Center for Advanced Ship and Deep-Sea Exploration, Shanghai 200240, China \\ * Correspondence: lizou@dlut.edu.cn (L.Z.); ycjiang@dlut.edu.cn (Y.J.)
}

Received: 15 July 2020; Accepted: 16 August 2020; Published: 20 August 2020

check for updates

\begin{abstract}
In recent years, the H-rotor vertical-axis turbine has attracted considerable attention in the field of wind and tidal power generation. After a series of complex spatiotemporal evolutions, the vortex shed from turbine blades forms a turbulent wake with a multi-scale coherent structure. An analysis of the wake characteristics of twin turbines forms the basis of array optimisation. This study aimed to examine the instability characteristics of a twin-turbine wake with two rotational configurations. The dynamic evolution characteristics of coherent structures with different scales in the wake were analysed via wavelet analysis. The results show that an inverse energy cascade process occurs after the high-frequency small-scale coherent structures induced by rotation lose their coherence. This self-organising characteristic is more apparent in the quasi two-dimensional wake of a forward-moving counter-rotating turbine (Array 1) than in that of a backward-moving counter-rotating turbine (Array 2). With greater organisation and coherence, the wake of Array 1 exhibits low-frequency instability characteristics dominated by a large-scale coherent structure. In addition, the signals reconstructed using wavelet transform show that asymmetric modes exist between low-frequency large-scale coherent structures. The experimental results provide a new perspective on the instability mechanism of twin-turbine wakes, as well as important data for numerical modelling.
\end{abstract}

Keywords: twin H-rotor vertical-axis turbines; wake; instability; wavelet transform

\section{Introduction}

Wind and tidal power generation technologies have advanced rapidly in recent years. Turbine wake is a type of unsteady turbulent flow. The wake effect exerts an important influence on the power generation efficiency of a turbine, fatigue life of a rotor, and stability of a power grid. Therefore, it is crucial to study the flow characteristics of turbine wake.

H-rotor turbine is the core equipment for vertical-axis wind power generation and tidal power generation [1,2]. Compared with the horizontal axis turbine, the vertical axis turbine can absorb energy in any direction of flow without complicated yaw system. The vertical axis turbine has a broad prospect in offshore wind power generation. The swept surface of a rotating vertical-axis H-rotor turbine (or horizontal axis turbine) blades is a cylinder (or disk). In many wake calculations, the turbine rotor can be described by a generalised actuator disk or actuator cylinder. To reduce the calculation for wake simulation, Rajagopalan et al. [3] used the CFD (Computational Fluid Dynamics) method to solve the generalised actuator disk rather than the dense blade grid and adopted the aerodynamic force acting on the wind turbine to the control equation. $\mathrm{Wu}$ [4] first described the nonlinear actuator disk model of 
a propeller using a formula. Although real numerical calculations were not conducted, the possibility of applying the actuator disk model to the numerical simulation of complex shapes was demonstrated. Conway [5] further studied the numerical analysis method. Madsen [6] was the first to propose a nonlinear actuator disk model for wind turbine aerodynamics and an actuator cylinder model to describe the flow field of vertical-axis wind turbines. Sørensen and Shen [7] introduced the actuator line method as an extension of the actuator disk method for non-uniform force. Shen and Zhang [8] extended the actuator line method to the actuator surface method and applied it to a vertical-axis wind turbine. The application of the method based on the actuation theory in the far wake region has yielded satisfactory results. However, research into the interaction between blade geometry and wake in this series of methods is still in progress, and the relevant theoretical knowledge requires further verification and supplementation.

Periodic vortex shedding is an instability that generally occurs in the wake of a typical blunt body, such as a cylinder or disk. This type of wake instability has also been reported in the wake of turbines. Medici and Alfredsson [9] detected a low-frequency peak in the wake spectrum. They determined it to be a manifestation of a blunt body wake mode that had evolved from the turbine's instability. Araya et al. [10] compared and analysed the wake of a vertical-axis turbine with the cylinder wake and linked them quantitatively.

Research on twin vertical-axis turbine arrays focuses mainly on the layout and output performance. Zanforlin and Nishino [11] used two-dimensional numerical simulation to study the aerodynamic performance of two counter-rotating vertical-axis turbines. It was observed that the overall power output of staggered turbines was less than that of parallel turbines. The decrease of power output depended on the direction of flow and the rotation configurations. Chen et al. [12] used a two-dimensional SST $k-\omega$ model based on DES simulation to study the power coefficients of two vertical-axis turbines by varying five factors. They observed that the five factors influenced the power factor to different levels (tip speed ratio $>$ inflow direction angle $>$ rotation direction $>$ spacing $>$ blade angle). The tip speed ratio had the greatest influence on the power output. Furthermore, the power coefficient of the two turbines was higher during reverse rotation. The power output of the two reverse rotating turbines was $9.97 \%$ higher than that of one turbine.

There are few reports on the study of the wake dynamic characteristics of twin vertical-axis turbines. Lam and Peng [13] studied the wake characteristics of a twin vertical-axis turbine with a spacing of $1 D$, through wind tunnel experiments ( $T / D=2 ; T=$ distance between the two centres; $D=$ diameter). It was observed that the method of reverse rotation aided the wake recovery. In particular, they observed that a pair of counter-rotating vortices in the wake evolution process also contributed to the wake recovery. Subsequently, they put forward two types of turbine array arrangement. In addition, there are few reports on the instability characteristics of the wake evolution of twin vertical-axial turbines. However, there have been substantial achievements in the study of the flow around two parallel cylinders. Zdravkovich and Pridden [14] divided the flow around two parallel cylinders into three main flow patterns according to the influence of the distance ratio on the wake: single blunt body mode $(1<T / D<1.1-1.2)$; asymmetric mode $(1.1-1.2<T / D<2-2.5)$, and symmetric mode $(T / D>2-2.5)$. There were biased flow patterns in the asymmetric mode region (Alam et al. [15], Zhou et al. [16], $\mathrm{Xu}$ et al. [17]). When the fluid flowed between two parallel cylinders, a random oscillating clearance flow was formed between the two cylinders. When the clearance flow deviated to one of the cylinders, the width of the wake became narrow and the vortex shedding frequency and drag coefficient increased. Simultaneously, the wake of the other cylinder widened and the vortex shedding frequency and drag coefficient reduced.

A relationship exists between the wake of a $\mathrm{H}$-rotor vertical-axis turbine and the cylinder wake. Furthermore, the two differ owing to the turbine rotation and vortex shedding by the blades. The differences and relationships between the wake characteristics of the two need to be explored further. Particularly, the wake instability, which occurs in the flow around two parallel cylinders, has rarely been reported in studies of the wake evolution of twin vertical-axial turbines. Therefore, wind tunnel 
tests are conducted to examine the space-time evolution characteristics of the wake of counter-rotating twin turbines with two rotational configurations, particularly the instability characteristics of the wake. The results of previous experiments [18] showed that with the increase of solidity (for example, the chord length remained unchanged at $81 \mathrm{~mm}$, and the number of blades changed from three to five), the vertical axis turbine presented a low-frequency instability mode characterized by large-scale coherent structure dominating wake development. At $5 D$, the five-blade turbine evolved into a low-frequency instability mode, while the wake of the three-blade turbine was still dominated by the rotation effect. In this study, wavelet analysis is also used to investigate wake instability of twin three-blade turbines.

The remainder of this paper is organised as follows: Section 2 introduces the experimental methods. Section 3.1 analyses the time-averaged characteristics of a forward-moving counter-rotating turbine (Array 1), describes the temporal and spatial evolution characteristics, and reveals the asymmetric modes between large-scale structures in the wake. Section 3.2 presents the time-averaged characteristics, evolution characteristics, and self-organisation characteristics of the wake of a backward-moving counter-rotating turbine (Array 2) and a comparative analysis with the wake of Array 1. Finally, Section 4 summarises the paper.

\section{Methods}

\subsection{Wind Tunnel and Twin Turbine}

The test section of the wind tunnel had a length of $18 \mathrm{~m}$, width of $3 \mathrm{~m}$, and height of $2.5 \mathrm{~m}$ (Figure 1: left side). The turbulence intensity at $6 \mathrm{~m}$ away from the entrance of the test section was $0.3 \%$ when the wind speed was $7.7 \mathrm{~m} / \mathrm{s}$. The distance between the centres of the two turbines was 1.2 times the diameter of the turbine $(T / D=1.2)$. The midpoint $(O)$ of the line between the geometric centre points of the two turbines' cross-sections coincided with the centre point of the cross-section of the test section.


Figure 1. H-rotor turbines in the wind tunnel.

Three blades with the NACA 0018 airfoil section and a pair of 2-mm-thick struts constituted the $\mathrm{H}$-shaped rotor of the turbine (Figure 1: right side). The chord length of the blade was $81 \mathrm{~mm}$, and the installation angle between the blade and strut was $0^{\circ}$. The diameter of the turbine $(D)$ was $600 \mathrm{~mm}$, 
and the height was $810 \mathrm{~mm}$. The two turbines were combined in different rotational directions to form two arrays (see Table 1). The direction of rotation of the turbine was observed from above.

Table 1. Description of the two arrays studied.

\begin{tabular}{ccc}
\hline Array \# & Turbine 1 & Turbine 2 \\
\hline 1 & Anticlockwise rotation & Clockwise rotation \\
2 & Clockwise rotation & Anticlockwise rotation \\
\hline
\end{tabular}

The limited size of the wind tunnel would produce a blocking effect. The following formula is provided for the solid block ratio:

$$
B=\frac{\text { rotor frontal area }}{\text { test section area }}
$$

According to Formula (1), the solid blocking ratio was $13 \%$, just above $10 \%$. The blocking effect is an unavoidable problem in wind tunnel test. However, when the numerical calculation is compared with the test data, the wind tunnel measurement environment can be well reproduced [19]. Therefore, there was no blocking correction in this study. The blocking correction can refer to the correction method of Savonius vertical-axis wind turbines in reference [20]. The wake measurement area of this test was controlled to within five times of the turbine diameter $(5 D)$, to minimise the wake blockage effect.

\subsection{Measurements}

The cobra probe used for wake measurement was a series 100 model manufactured by TFI. The sampling frequency selected in this experiment was $100 \mathrm{~Hz}$, which satisfied the scale requirements of the study. The measurement area is shown in Figure 2. Because the two turbines were arranged symmetrically, only the wake information of Turbine 1 was collected in the test. The sampling time of each measuring point was $30 \mathrm{~s}$.

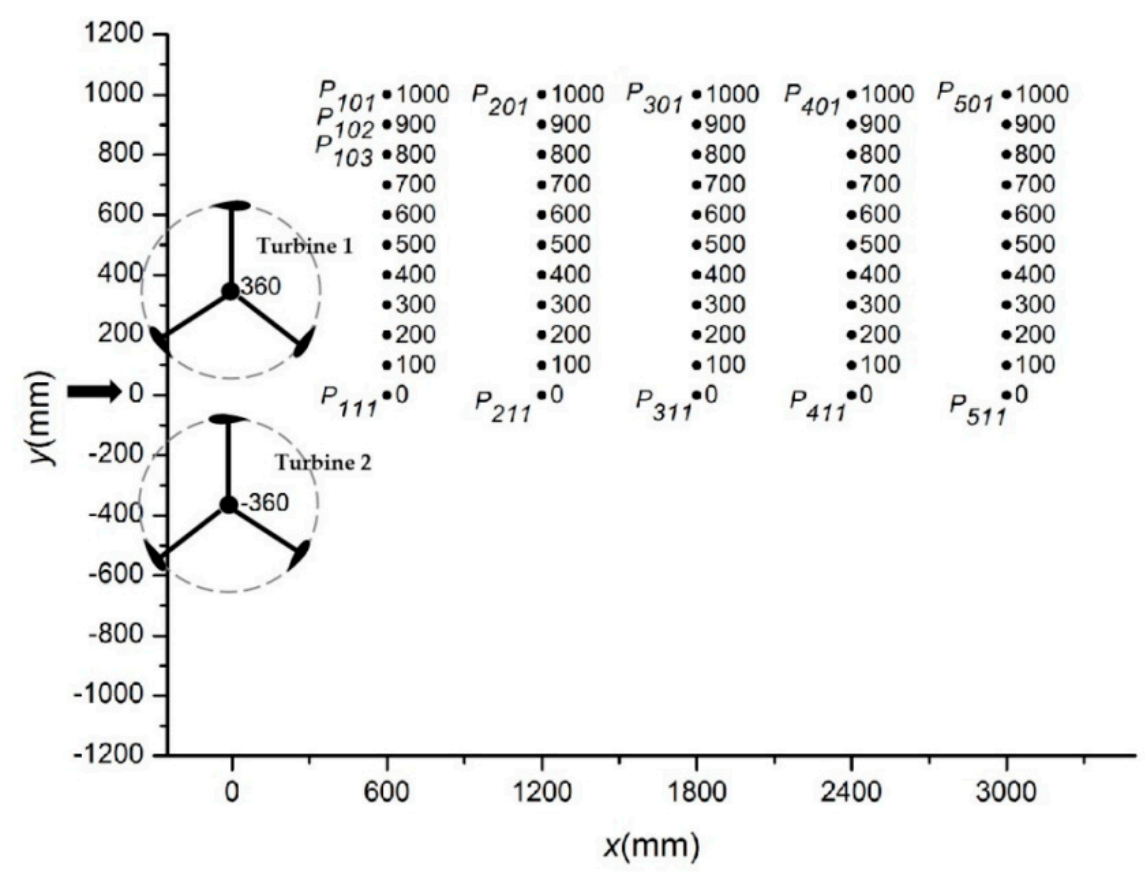

Figure 2. Measurement points at $z=0$. 
The system errors $\left(E_{S}\right)$ were estimated by measuring the flow inconsistencies in the empty tunnel. The uncertainty $\left(E_{S x}\right)$ of the system errors in the $x$-direction was estimated by measuring the streamwise velocity at $P_{111}, P_{211}, P_{311}, P_{411}$, and $P_{511}$. The uncertainty $\left(E_{S y}\right)$ of the system errors in the $y$-direction was determined by collecting the streamwise component of the 21 points from $P_{301}$ to $P_{321}$. The uncertainty $\left(E_{R}\right)$ of the random errors was determined by repeatedly collecting the streamwise component at $P_{304}$ in the turbine wake. The overall uncertainty was determined according to the following formula provided in reference [13]:

$$
E_{T}=\sqrt{\left(E_{R}\right)^{2}+\left(E_{S x}\right)^{2}+\left(E_{S y}\right)^{2}},
$$

The measurements yielded the following: $E_{S x}=0.9 \%, E_{S y}=1.2 \%$, and $E_{R}=0.4 \%$. Thus, the overall uncertainty was $1.6 \%$.

Moreover, the turbine torque output at different rotation speed was measured. The torque output curves are given in Figure A1 of the Appendix A. The wake of a turbine depends on its operating state [21]. The operating state at $500 \mathrm{rpm}$ was selected as a typical condition to collect wake information.

\subsection{Wavelet Analysis Method}

The wavelet coefficient formula of a signal $f(t)$ can be expressed as

$$
T(a, b)=\left(f(t), \psi_{a, b}(t)\right)=\frac{1}{\sqrt{a}} \int_{-\infty}^{+\infty} f(t) \psi^{*}\left(\frac{t-b}{a}\right) d t,
$$

$T(a, b)$ is called the wavelet transform coefficient, and $t$ is the time variable. Here, $a$ is the expansion variable, and $b$ is the translation variable. $\Psi$ is the mother wavelet, and $\Psi^{*}$ is the conjugate of $\Psi$. A Morlet wavelet [22] was used as the mother wavelet in this study. Both wavelet and Fourier are integral transforms. Expanding a function under a wavelet basis function implies the projection of a function onto a two-dimensional time-scale plane.

Wavelet analysis is an important method for turbulence research. It compensates for the spectrum analysis' dependence on the number of samples and time interval. It can be used for a localisation analysis. In a wavelet analysis, the Fourier transform basis function is replaced with a 'wavelet function'. It has a good time-frequency analysis function, can conduct multi-scale and multi-resolution analyses of time series signals, and is an effective tool for analysing the energy distribution of various frequency component signals and for extracting local information. Moreover, the variation in the wavelet energy spectrum intensity with time and frequency can reveal other physical mechanisms [23].

\section{Results and Discussion}

\subsection{Forward-Moving Counter-Rotating Turbine}

For Array 1, Turbine 1 rotates anticlockwise, and Turbine 2 rotates clockwise (Figure 3b). The blades passing through the intermediate clearance between the two turbines always move forward $(x+)$ in the wind direction.

\subsubsection{Time-Averaged Wake Characteristics}

Figure $3 a$ shows the normalised time-averaged streamwise velocity distribution. Figure $3 b$ is the interpolated cloud chart of Figure 3a. In this test, the free inflow $\left(U_{0}\right)$ was maintained at $7.7 \mathrm{~m} / \mathrm{s}$. Both the turbines rotated at $500 \mathrm{rpm}$. Figure 3 shows that there is a velocity deficit region downstream of the rotating rotor, owing to momentum extraction and the blocking effect. The speed is not immediately reduced to the maximum attenuation value when the free inflow passes through the turbine rotor. It first moves downstream for a certain distance, attains the maximum speed attenuation value, and then recovers gradually. The interaction between the clearance flow and wake flow results in the development of the intermediate shear layer, whereas the interaction between the free inflow 
and wake flow results in the development of the lateral shear layer. Figure 3 shows that the shear layer expands continuously in the process of developing downstream. In addition, wake excursions induced by the rotor's rotation can be observed. The above characteristics are in accordance with the literature [24-26].

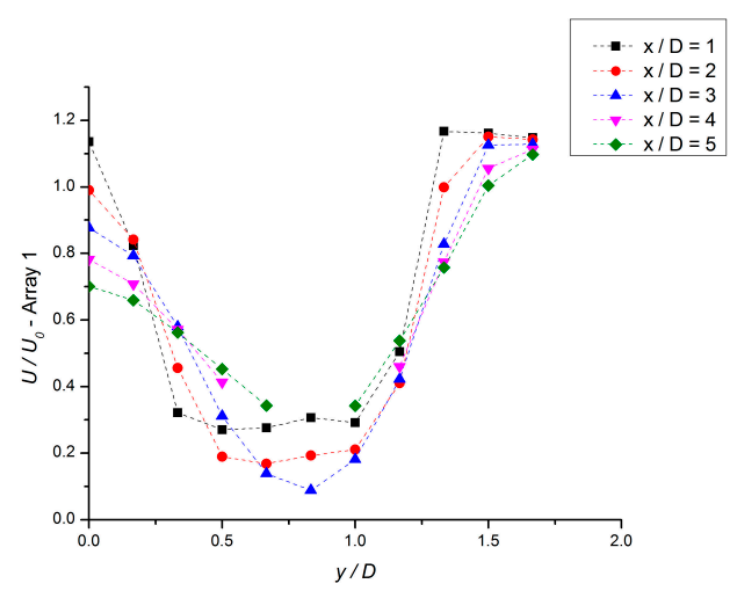

(a)

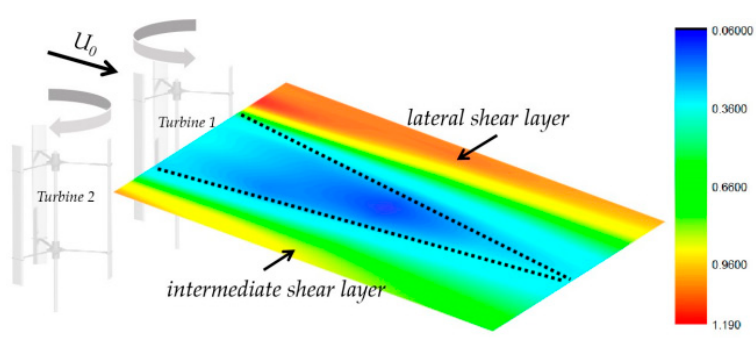

(b)

Figure 3. Time-averaged streamwise velocity distribution (a) and interpolation nephogram (b) of wake of Array 1.

In particular, the velocity information of a few measuring points at $4 D$ and $5 D$ in Figure $3 a$ is absent. There are a large number of zero values in the velocity time series measured at $4 D$ and $5 D$. The time series defaults to zero because the cobra probe cannot capture the return velocity. Although the probe can output the time-averaged velocity information, it cannot reflect the flow fact. Therefore, the velocity information of the re-circulation region at $4 D$ and $5 D$ in Figure $3 a$ is not analysed statistically. The re-circulation region had been addressed in accordance with reference [27]. Notably, the negative velocity information of the re-circulation region is not reflected in Figure $3 \mathrm{~b}$. This is because the cloud images in this paper are interpolation cloud images. The cloud charts in this paper are only for illustration.

Figure 4 shows the distribution of normalised normal Reynolds stress $\overline{u u} / U_{0}^{2}$ and the interpolated cloud chart. The maximum value appears at $1 D$ of the intermediate shear layer, where a strong shear is formed between the clearance flow and wake flow. The secondary maximum value appears in the lateral shear layer downstream of the re-circulation region. The maximum values are concentrated in the shear layer with a sharp velocity gradient. The shear layer contains coherent structures of different scales. These coherent structures develop and brake, which causes the low-speed fluid in the wake to mix with the high-speed free inflow outside. Thereby, the momentum of the external free inflow is transferred to the wake, which causes the wake area and shear layer to expand gradually. Next, the wavelet transform is used to determine the dynamic evolution characteristics. 


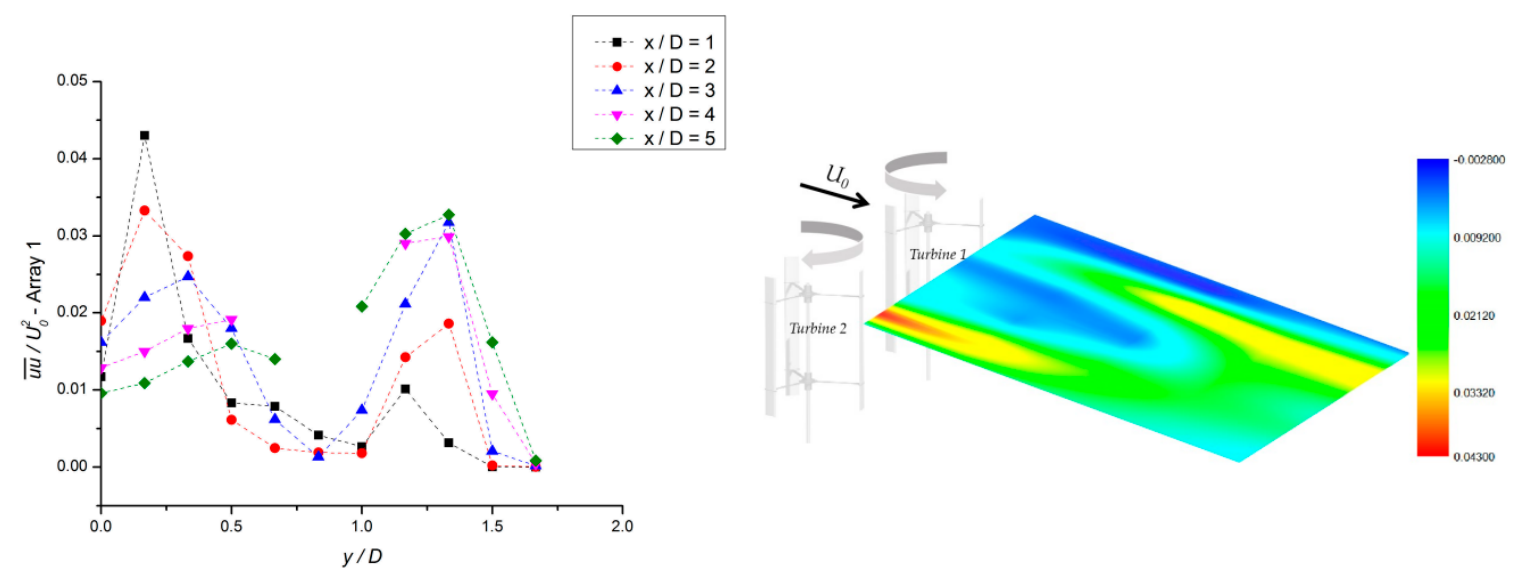

(a)

(b)

Figure 4. Normal Reynolds stress distribution (a) and interpolation nephogram (b) of wake of Array 1.

\subsubsection{Dynamic Characteristics of Evolution}

Figure $5 b-d$ shows the wavelet energy spectrum of the measuring points in the lateral shear layer. Blade vortex shedding ( $3 f$ ) dominates the wake development at $P_{103}$ (Figure $5 b$ ). In addition, a coherent structure with a lower frequency appears, which indicates that the blade vortex shedding begins to lose coherence gradually when the wake reaches $1 D$. As the wake continues to develop downstream and reaches $3 D P_{303}$ (Figure $5 \mathrm{c}$ ), the blade vortex shedding breaks completely and disappears. However, the coherent structure induced by rotation still dominates the wake development. The coherent structure with the rotating frequency $f$ as the dominant frequency can be observed clearly. Meanwhile, the energy of the low-frequency coherent structure continues to increase, whereas that of the high-frequency coherent structure continues to decrease. When the wake coherent structure develops to $4 D P_{402}$ (Figure $5 \mathrm{~d}$ ), the high-frequency small-scale coherent structure induced by rotor rotation breaks and disappears. Furthermore, the low-frequency coherent structures are relatively stable and independent, thereby dominating the wake development. The low-frequency coherent structure of $4 D$ is the result of the evolution of the coherent structure of $3 D$. In the evolution process, the dominant frequency of the coherent structure decreases gradually, and its scale continues to increase.

Figure $6 \mathrm{~b}, \mathrm{c}$ is the wavelet energy spectrum of the measuring points in the intermediate shear layer. The blade vortex shedding has broken completely and disappeared at $1 D P_{110}$, unlike in the case of the lateral shear layer. The small-scale coherent structure with frequency $f$ induced by rotation dominates the wake development. A low-frequency large-scale coherent structure appears simultaneously. At $2 D$ $P_{210}$, the coherence of the low-frequency large-scale structures increases gradually, whereas most of the small-scale coherent structures induced by rotation have broken. 


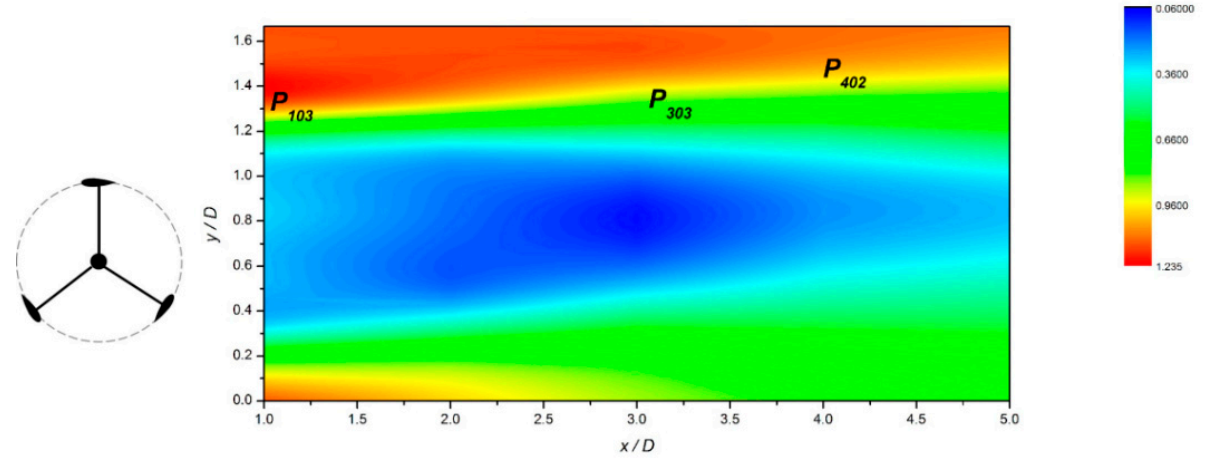

(a)

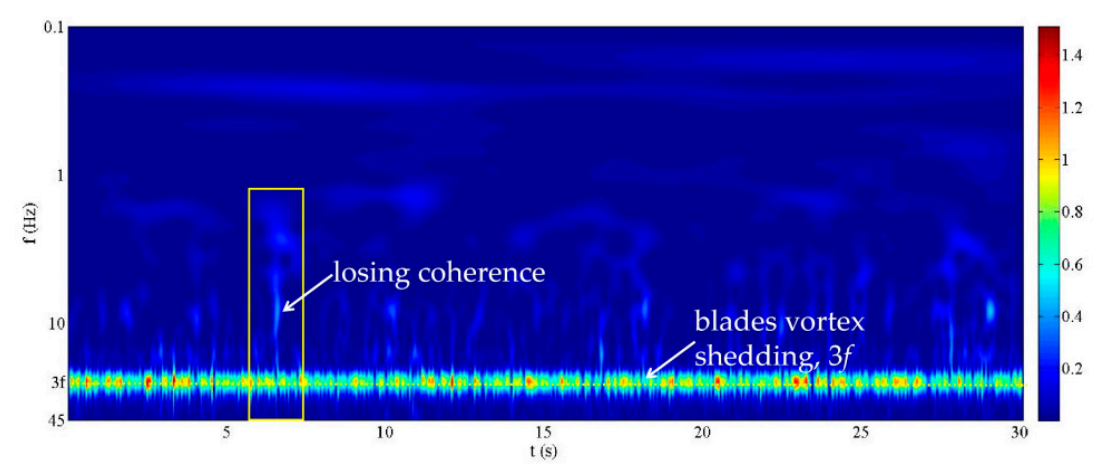

(b)

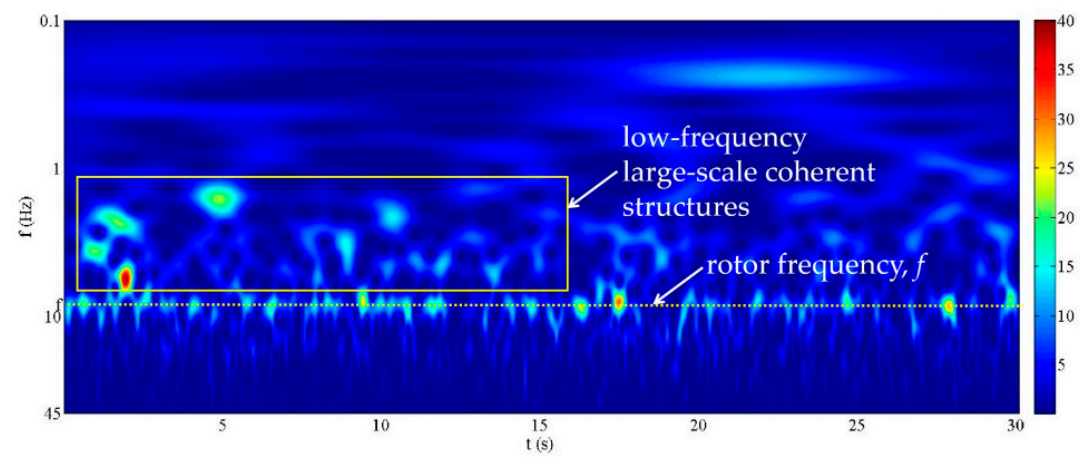

(c)

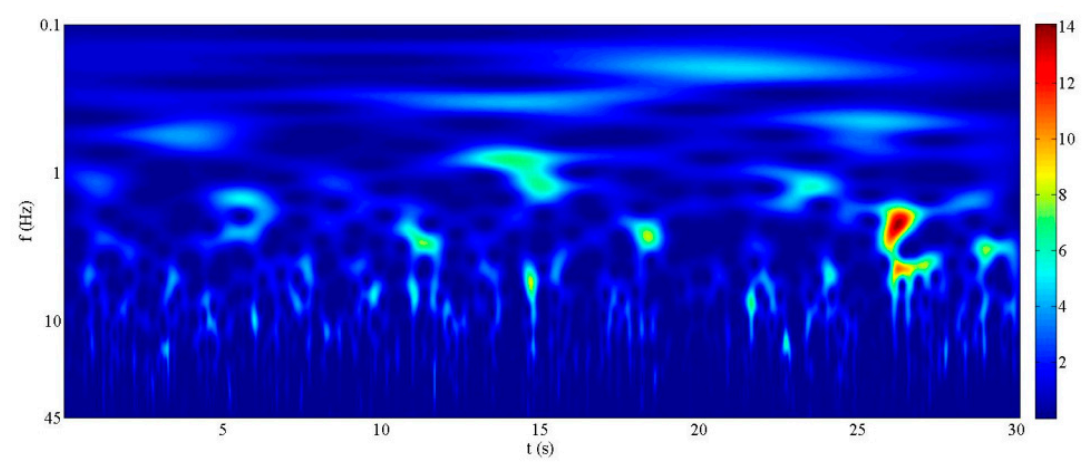

(d)

Figure 5. Wavelet transform analysis of time series at different measuring points in the lateral shear layer: (a) schematic diagram, (b) $P_{103}$, (c) $P_{303}$, (d) $P_{402}$. 


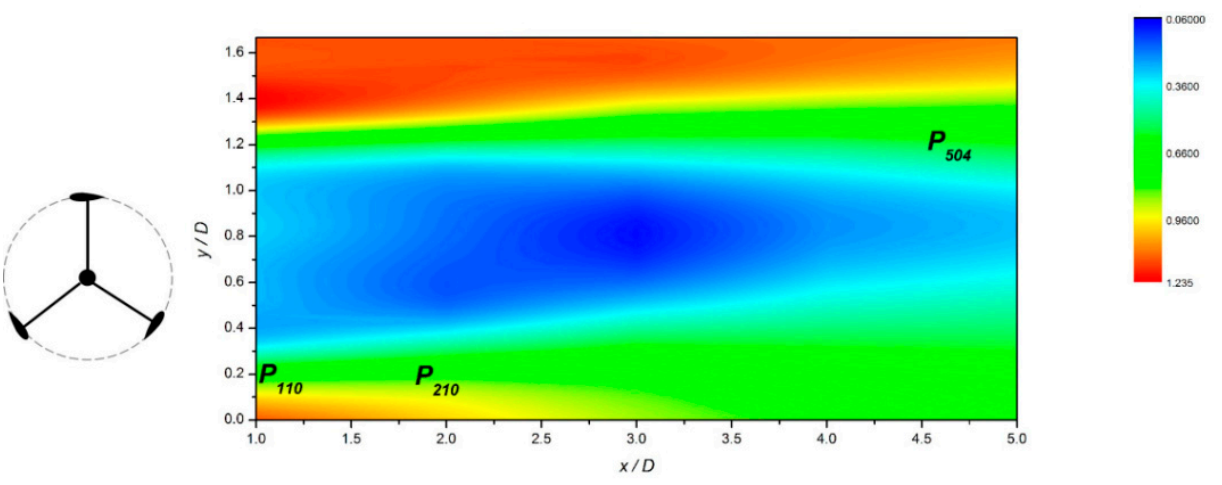

(a)

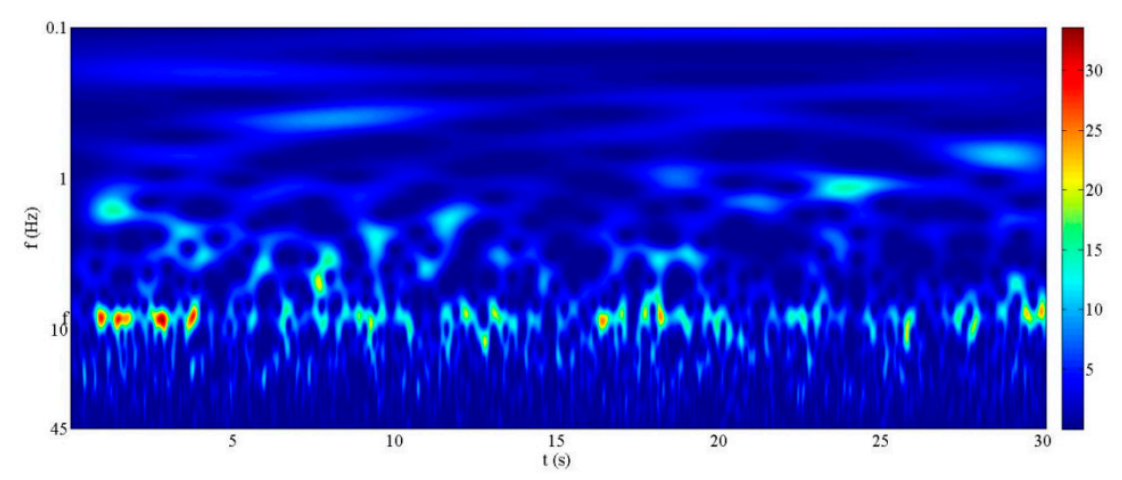

(b)

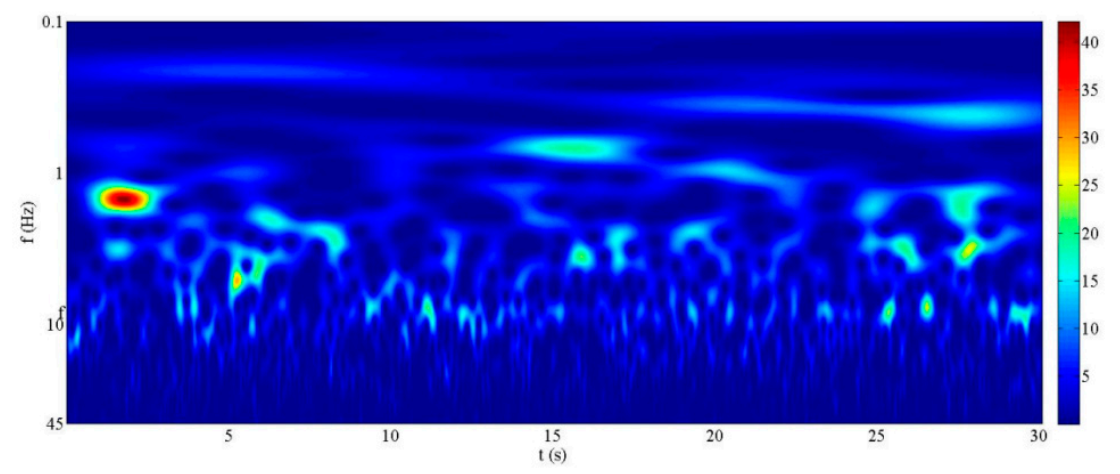

(c)

Figure 6. Wavelet transform analysis of time series at different measuring points in the intermediate shear layer: (a) schematic diagram, (b) $P_{110}$, (c) $P_{210}$.

The direction of blade movement through the two turbine intervals of Array 1 is identical to that of the incoming flow. Furthermore, the direction of blade movement on both the sides is opposite to that of the incoming flow. This rotational configuration promotes the development of clearance flow, which accelerates the loss of coherence of blade vortex shedding and the occurrence of shear layer instability. This rotational configuration also causes the wakes of the two turbines to shift outwards relative to each other. A strong shear is produced by the outward offset wake and the free inflow. In the lateral shear layer, the blade vortex shedding loses coherence in $2 D$ under strong shear. As the blade vortex shedding loses coherence, the rotation induction effect disappears gradually and the energy of the high-frequency small-scale coherent structure decreases gradually. Then, the dominant frequencies (3D and 4D) of the wake coherent structure gradually decrease and the scale gradually increases. The time-averaged wake velocity distribution (Figure 3a) indicates the presence of a re-circulation region at $4 D$ and $5 D$. In addition to the re-circulation region, $5 D P_{504}$ (Figure 6a) is selected, and the wavelet energy spectrum 
of its time series signal is shown in Figure 7. As is evident from Figure 7, as the wake development proceeds, the organisation of the coherent structure is enhanced, and the energy is concentrated in the low-frequency range of $2-3 \mathrm{~Hz}$. The constant Strouhal number is between 0.16 and 0.23 with respect to $D$. The low-frequency instability characteristic of the large-scale coherent structure dominating the wake development was mentioned in reference [10]. Therein, the wake of a vertical-axis turbine evolved into a blunt body wake mode, and the Strouhal number was approximately 0.26 . However, the Strouhal number in this study fluctuates in a narrow frequency band.

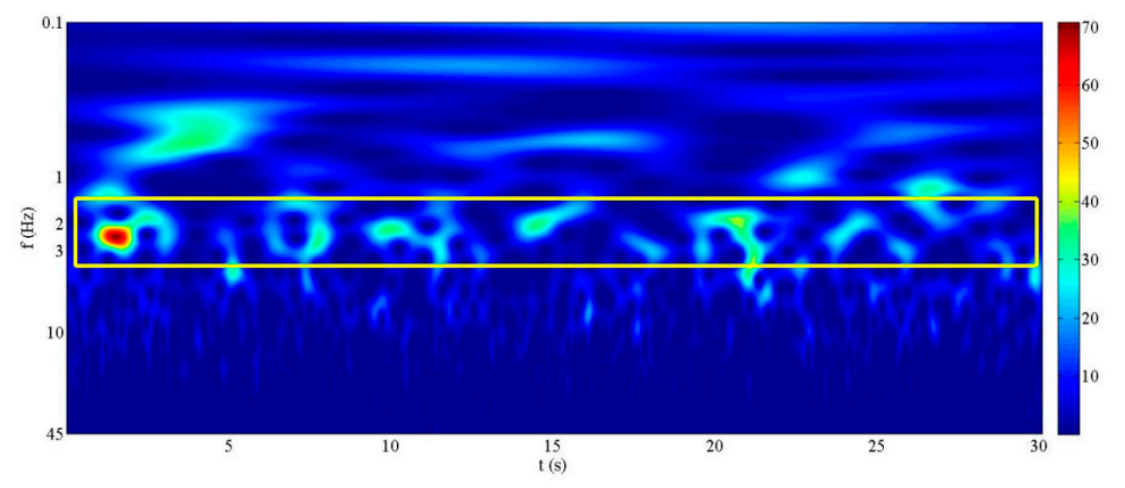

Figure 7. Wavelet transform analysis of time series at $P_{504}$.

As mentioned earlier, the rotating configuration of Array 1 causes the two turbines' wakes to diverge away from each other. This promotes the development of clearance flow. It can be inferred that the clearance flow that is biased towards a turbine causes the wake on this side to narrow with an increase in the shedding frequency. Simultaneously, the clearance flow causes the wake on the other side to widen with a decrease in the shedding frequency. That is, there are asymmetric modes between the large-scale coherent structures in the wake evolution process of the twin turbines in Array 1. Next, wavelet decomposition and reconstruction are applied to demonstrate this flow pattern.

\subsubsection{Asymmetrical Mode}

Figure 8 is a local enlarged image of Figure 7 , which is a typical wavelet energy spectrum of the scale of dynamic variation, for the period 13-19 s. This figure also reflects the intermittent process of turbulence effectively. The top of Figure 9 is the original time series signal of $P_{504}$, and the frequency range of the original signal is $0-48.4 \mathrm{~Hz}$. The bottom graph in Figure 9 shows the fifth layer high frequency component $u_{5}$ after wavelet decomposition and reconstruction of the original signal; $u_{5}$ has a frequency range of $1.5-3 \mathrm{~Hz}$. In this study, the db3 wavelet is used for the decomposition and reconstruction.

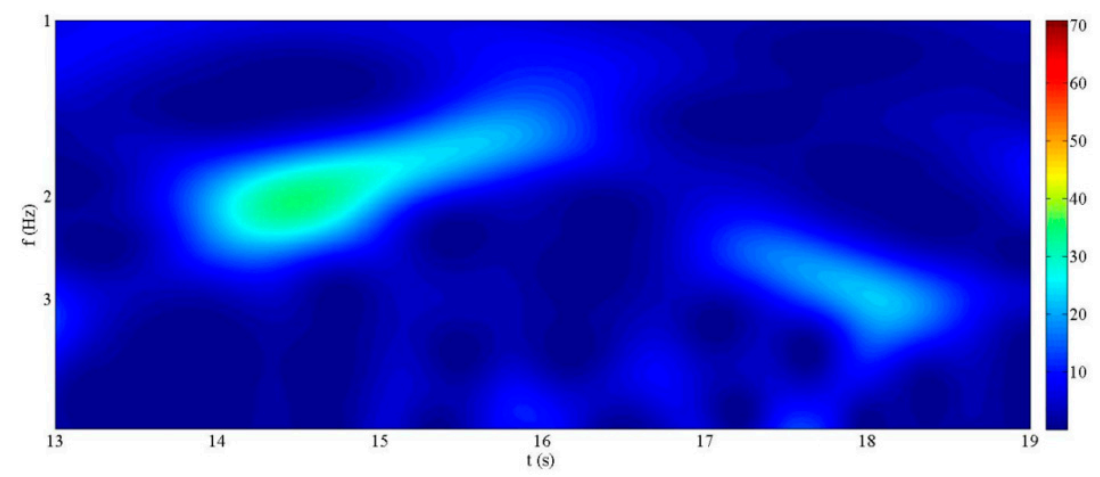

Figure 8. The 13-19 s local graph of Figure 7. 

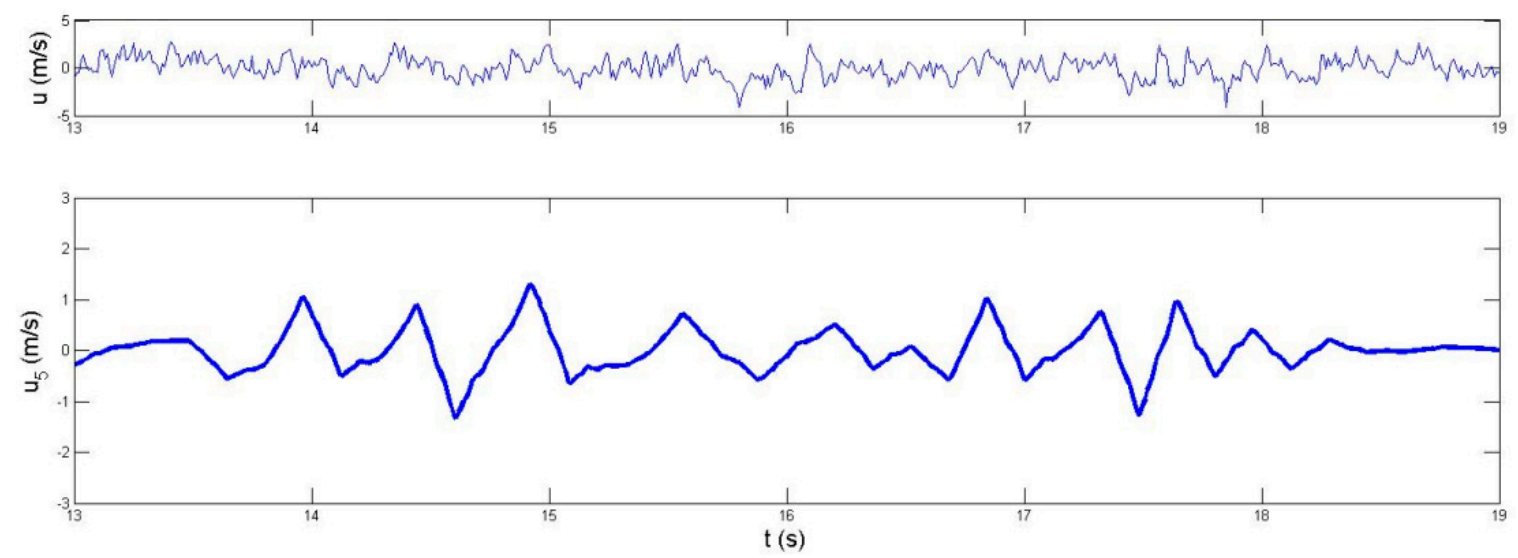

Figure 9. Original time series signal of $P_{504}$ (top) and the reconstructed signal (bottom).

As is evident from Figure 8, the frequency of the coherent structure is mainly about $2 \mathrm{~Hz}$ between 14 and $15 \mathrm{~s}$. Furthermore, it is in a state of continuous reduction. The dominant frequency of the coherent structure continues to decrease in 15-16 s and attains $1 \mathrm{~Hz}$. That is, in 14-16 s, the coherent structure is in a mode where the dominant frequency decreases continuously. The continuous decrease in the dominant frequency implies that the scale of the coherent structure continues to increase, i.e., the wake continues to widen. The reconstructed velocity time series (Figure 9: bottom) also shows the dynamic process of the gradually increasing cycle in 14-16 s. The periodicity disappears in 16-17 s. Another mode appears in the coherent structure in 17-18 s (Figure 8). The dominant frequency of the coherent structure begins to increase, and the scale decreases continuously, i.e., the wake continues to narrow.

The previous evolution process reveals that the low-frequency large-scale coherent structure dominates the wake development of Array 1 . In addition, the low-frequency instability fluctuates in a narrow frequency band. Asymmetric modes exist between the large-scale coherent structures.

\subsection{Backward-Moving Counter-Rotating Turbine}

For Array 2, Turbine 1 rotates clockwise, and Turbine 2 rotates anticlockwise (Figure 10b). The direction of blade movement through the intermediate clearance of the two turbines is always backward ( $x$ is negative) against the wind.

\subsubsection{Time-Averaged Wake Characteristics}

As shown in Figure 10a,b, the degree of expansion of the intermediate shear layer in the wake of Array 2 is less than that of Array 1. This is because the two turbines' wakes in Array 2 are close to each other. The Reynolds normal stress in the intermediate shear layer (Figure 10c,d) first increases and then decreases.

The normal stress distribution of the lateral shear layer (Figure 10c,d) has the maximum value at $1 D$. Thereafter, it shows a weakening trend. This is because Turbine 1 rotates clockwise and the direction of blade movement through the lateral shear layer is identical to that of the incoming flow. The normal stress distribution trend of the lateral shear layer of Array 1 (Figure $4 a, b$ ) is contrary to that of Array 2 owing to the different rotational configurations. 


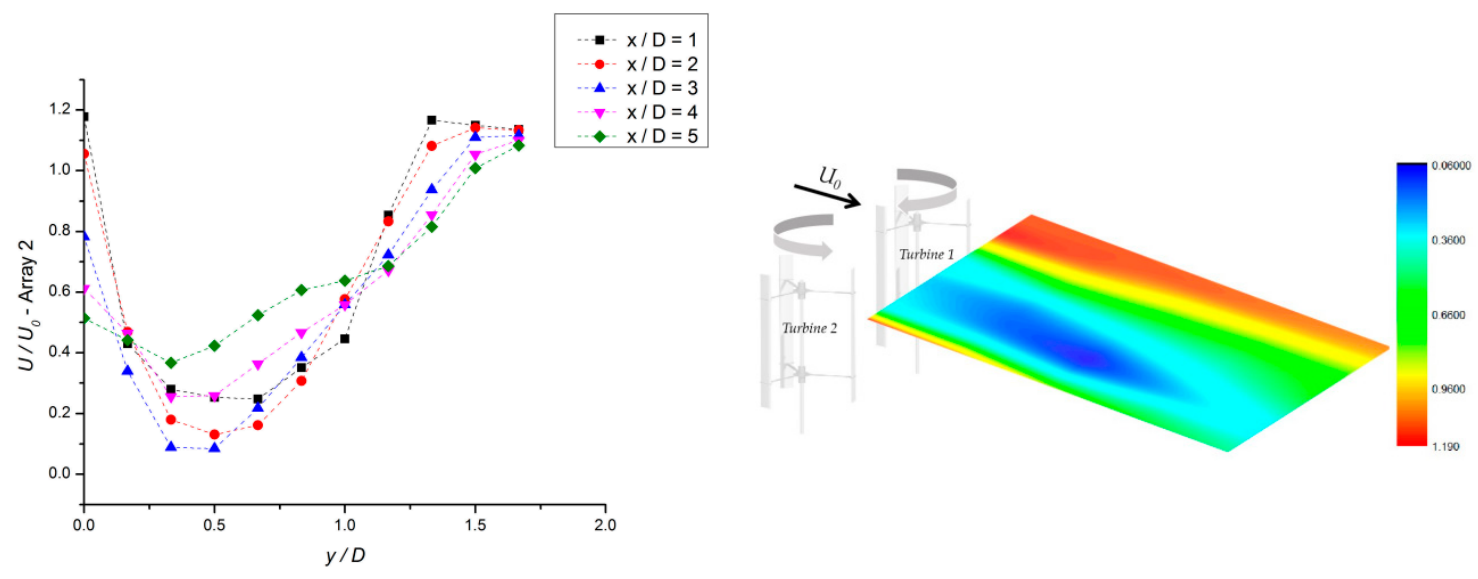

(a)

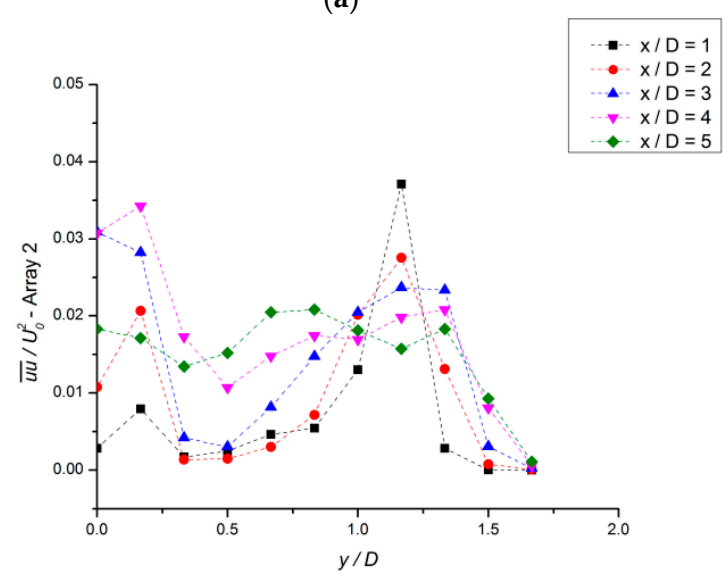

(c) (b)

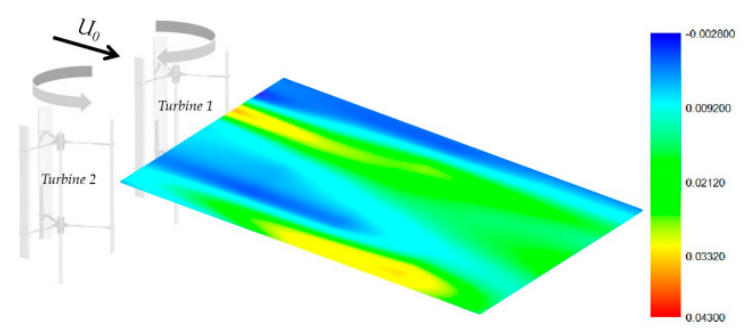

(d)

Figure 10. Time-averaged streamwise velocity distribution (a) and interpolation nephogram (b) of wake of Array 2. Normal Reynolds stress distribution (c) and interpolation nephogram (d) of wake of Array 2.

\subsubsection{Dynamic Characteristics of Evolution}

In the intermediate shear layer $1 D P_{110}$ (Figure 11b) of the wake of Array 2, the blade vortex shedding interaction increases. However, the wake development continues to be dominated by the blade vortex shedding with a frequency of $3 f$. However, the blade vortex shedding of Array 1 completely disappears at $1 D P_{110}$ (Figure 6b). At $3 D P_{311}$ (Figure 11c), the rotation induction effect still exists. While the wake of Array 1 is in $2 D$ (Figure 6c), most of the coherent structures with frequency $f$ have broken. At $5 D P_{503}$ (Figure 11d), although the energy of the low-frequency large-scale coherent structures has increased, their energy is not concentrated, and their organisation and coherence are not strong. In addition, the high-frequency small-scale coherent structure induced by rotation still exists.

It is evident from the evolution process of the coherent structure that the interaction between the lateral shear and free inflow is weaker than that of Array 1 because the wakes of the two turbines in Array 2 are close to each other. The high-frequency small-scale coherent structure passing through the lateral shear layer loses coherence after that of Array 1. This rotation configuration also hinders the development of clearance flow. The high-frequency small-scale coherent structure induced by rotation passing through the clearance also loses coherence after that of Array 1. In conclusion, the Array 2 turbine does not exhibit the low-frequency instability characteristic of the large-scale coherent structure dominating the wake development. 

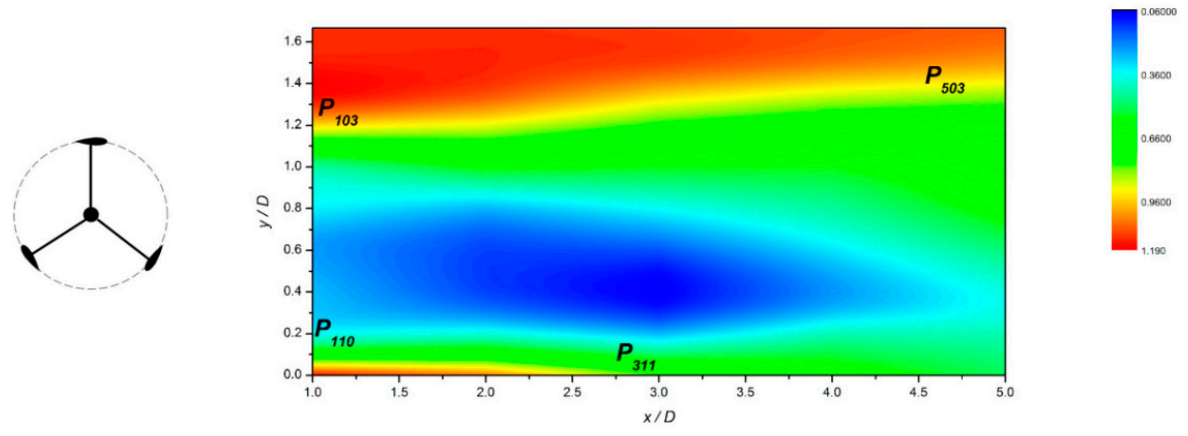

(a)

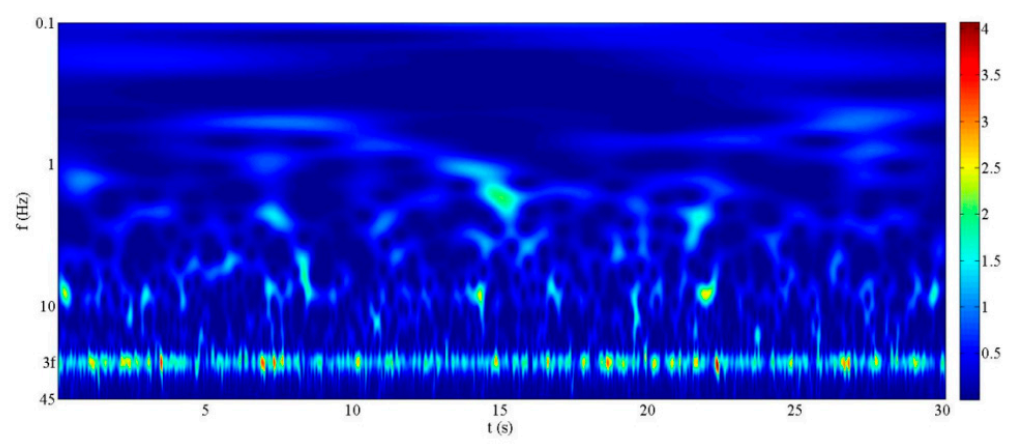

(b)

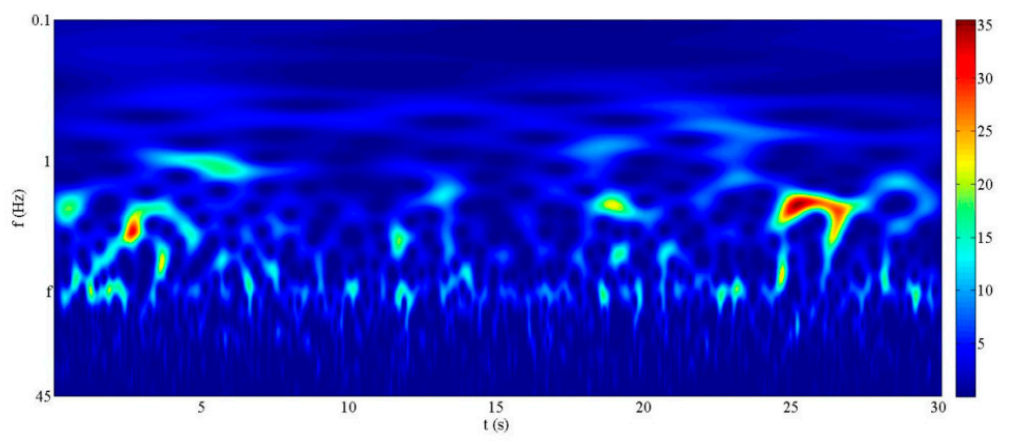

(c)

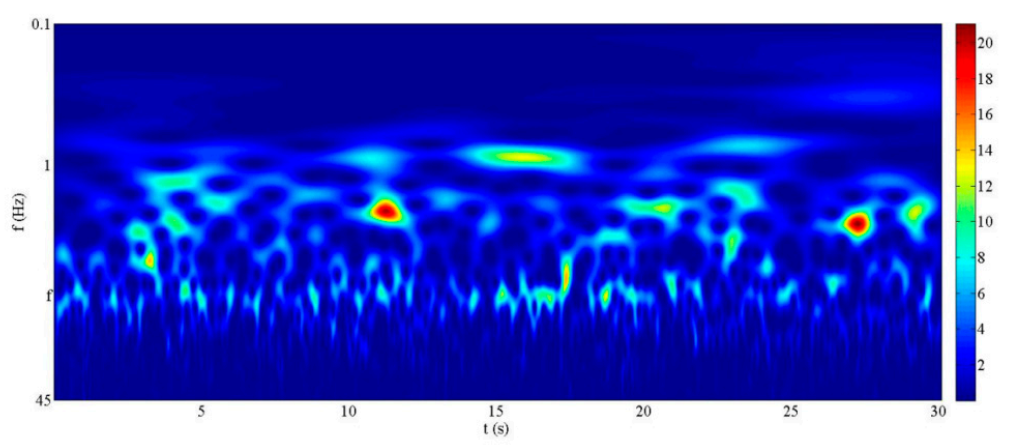

(d)

Figure 11. Wavelet transform analysis of time series at different measuring points in the shear layer: (a) schematic diagram, (b) $P_{110}$, (c) $P_{311}$, (d) $P_{503}$. 


\subsubsection{Self-organisation Characteristics}

Previous research has shown that the high-frequency small-scale coherent structures (frequency $3 f$ and $f$ ) induced by rotation dominate the wake development in the near wake. As shown in Figure $12 \mathrm{~b}$ (local part of Figure 12a), at $1 D P_{103}$ of the lateral shear layer of Array 2, the coherent structure with the frequency of $3 f$ gradually loses coherence and disintegrates. Thereby, it draws energy from the average flow and transfers it to the smaller scale vortex structure (down arrow in Figure 12b). This process is consistent with the energy cascade phenomenon. In addition, the vortex structures merge, the frequency decreases, and the scale increases (the up arrow in Figure 12b).

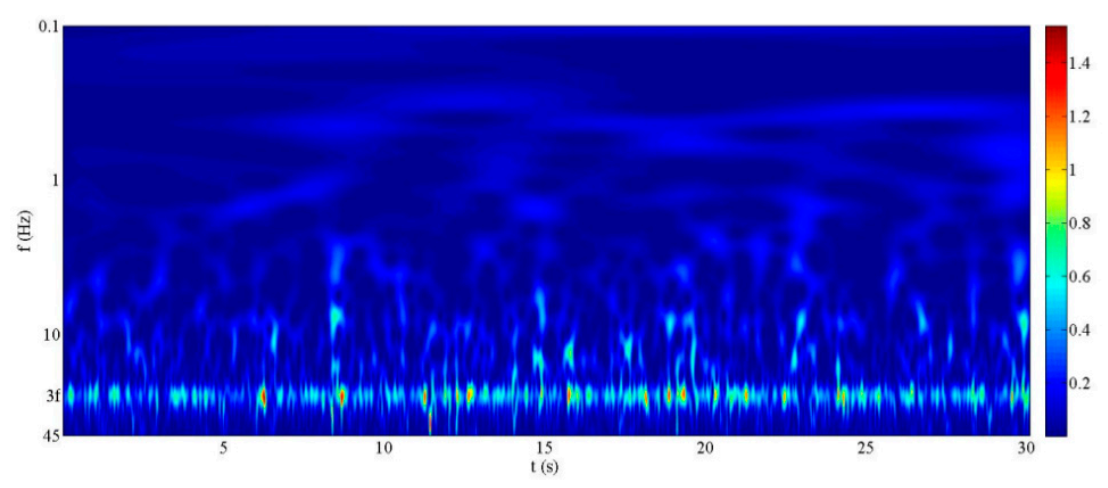

(a)

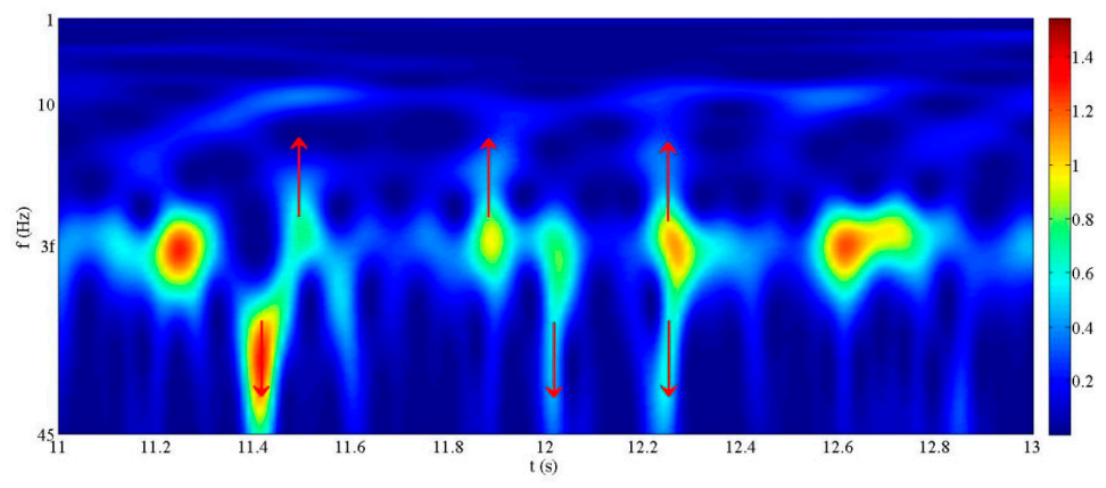

(b)

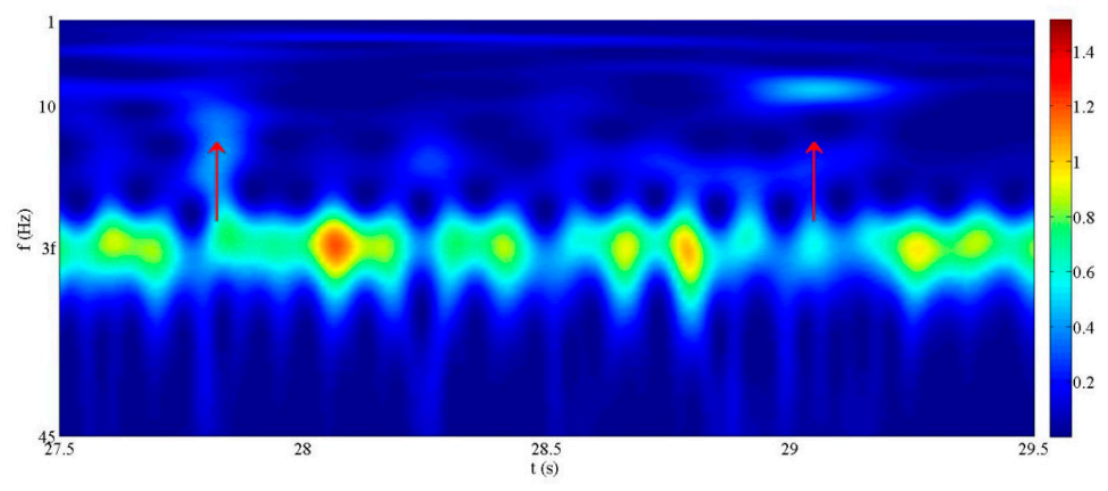

(c)

Figure 12. Wavelet transform analysis of time series at $P_{103}(\mathbf{a})$. Local parts of Figure 12a (b) and Figure $5 b(c)$. 
At $1 D P_{103}$ of the lateral shear layer of Array 1, the adjacent small-scale coherent structures interact with each other, the dominant frequency decreases gradually, and the scale increases gradually (the up arrow in Figure 12c). It is evident from the previous research that, as the wake continues to develop downstream, the organisation and coherence of large-scale coherent structures become stronger. Furthermore, the energy is gradually transferred to the low-frequency structure. Finally, at $5 D$ $P_{504}$ (Figure 7), the background small-scale coherent structure is very weak.

The above evolution process illustrates that there is an inverse energy cascade process in the wakes of Array 1 and Array 2 and that the inverse energy cascade process of Array 1 is more apparent. In [28], it was indicated for the first time that there is a series of inverse energy cascade processes in two-dimensional isotropic turbulence, i.e., the energy transferred from a small- to a large-scale structure in the development process of two-dimensional turbulence. Subsequently, many scholars studied two-dimensional turbulence by numerical simulation or experimental methods and further verified the existence of two-dimensional turbulent inverse energy cascade. It was observed that the adjacent small-scale vortices in the flow field interacted with each other and merged into larger vortices. Finally, one or several relatively stable self-organised large vortices with the same size as that of the physical domain could be formed. These are the self-organisation characteristics of two-dimensional turbulence [29-34]. In essence, turbulence is a completely three-dimensional nonlinear fluid motion. However, certain specific flows display apparent quasi two-dimensional characteristics [28]. This is mainly because the scale of such a flow in one direction is significantly smaller than that in the other two directions.

Figure 13 shows the profiles of the six components of Reynolds stress at $1 D$ and $5 D$ for the wakes of Array 1 and Array 2. The three components of Reynolds normal stress $(\overline{u u}, \overline{v v}, \overline{w w})$ in the wake of Array 1 (Figure 13a) are almost identical at $1 D$, whereas the Reynolds shear stress component $(\overline{u w}, \overline{v w})$ is essentially zero. For Array 2 (Figure 13b), the streamwise direction and vertical direction in the $1 D$ lateral shear layer have an apparent correlation $(\overline{u w})$, and a shear effect is observable. That is, the three-dimensional characteristics of the wake of Array 2 are more apparent than those of Array 1 at $1 D$. This is true at $5 D$ as well (Figure $13 c, d$ ).

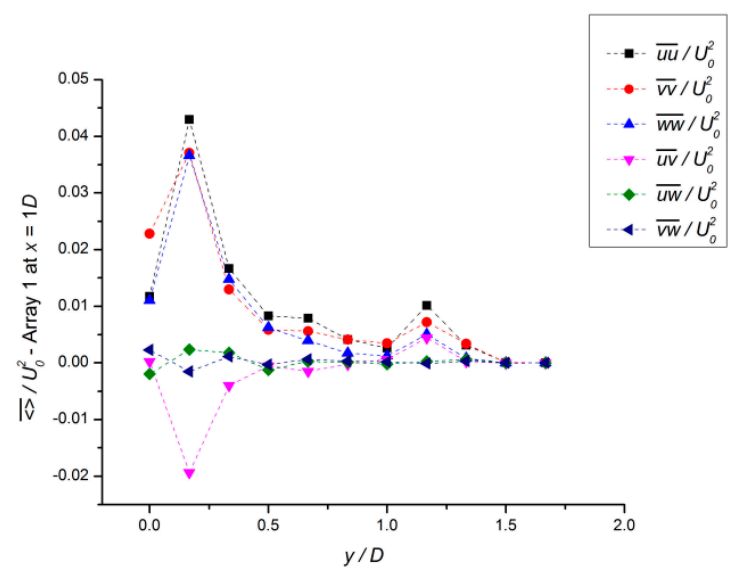

(a)

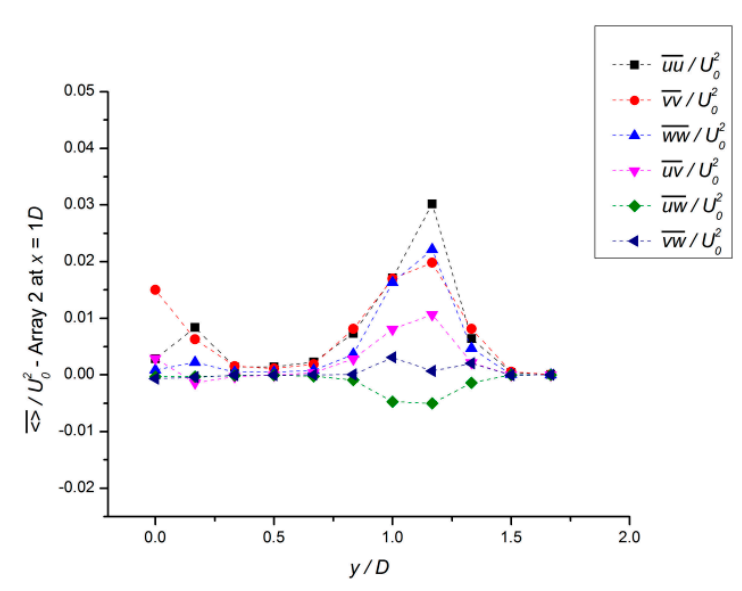

(b)

Figure 13. Cont. 


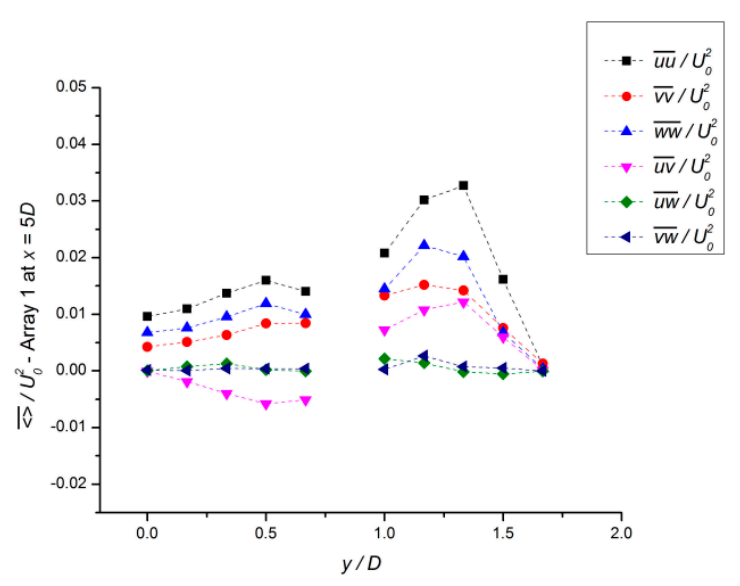

(c)

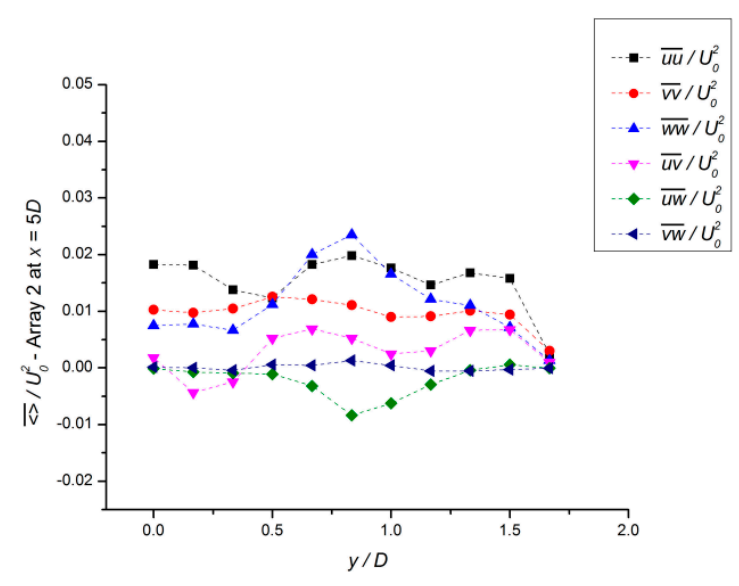

(d)

Figure 13. The profiles of the six components of Reynolds stress at $1 D(\mathbf{a}, \mathbf{b})$ and $5 D(\mathbf{c}, \mathbf{d})$ for the wakes of Array 1 and Array 2.

In the quasi two-dimensional wake of Array 1, the small-scale structure continuously transfers energy to the large-scale structure in accordance with the principle of inverse energy cascade. Therefore, the wake flow is constantly modified to carry more energy. However, in the three-dimensional wake of Array 2, the coherent structure continuously absorbs energy from the free inflow to transfer it to the smaller turbulence structure. Finally, the energy is dissipated from the smallest turbulence vortex and is converted to the internal energy of the fluid. As a result, the time-averaged streamwise velocity profile of Array 2 (Figure 10a) is flatter than that of Array 1 (Figure 3a).

\section{Conclusions}

This paper focuses on the wake instability characteristics of two types of counter-rotating twin turbines with a spacing of $0.2 D(T / D=1.2)$. The rotational configuration of Array 1 (forward-moving counter-rotating turbine) causes the formation of strong shear in the lateral shear layer and promotes the development of clearance flow. This, in turn, results in the formation of strong shear in the intermediate shear layer. The high-frequency small-scale coherent structure in the near wake induced by rotation gradually loses coherence in the intermediate and lateral shear layers. This causes the shear layers to become unstable. The instability mechanism is induced earlier in the wake of Array 1 than it is in the wake of Array 2 (backward-moving counter-rotating turbine). After the high-frequency small-scale coherent structure loses coherence, energy is transferred to the smaller-scale coherent structure according to the energy cascade principle. Meanwhile, the small-scale coherent structure transfers energy to the low-frequency coherent structure to form a self-organised large-scale coherent structure. This type of self-organisation characteristics is more apparent in the quasi two-dimensional wake of Array 1 than that in the wake of Array 2. With the development of the wake downstream, the organisation, coherence, and scale of the low-frequency structure in the wake of Array 1 is enhanced gradually. Ultimately, low-frequency large-scale coherent structures dominate the wake development. In addition, there are asymmetric modes between the large-scale coherent structures in the wake of Array 1, which causes the Strouhal number to fluctuate between 0.16 and 0.23 .

These results have certain reference significance for studying wake flow mechanism and optimizing turbine array layout. The self-organised large-scale coherent structure has a positive role in promoting turbulent mixing and entrainment, but also has a negative effect on fatigue load and power fluctuation. Further studies can consider the flow control of wake large-scale coherent structure.

Author Contributions: Conceptualization, K.W.; data curation, K.W.; formal analysis, K.W.; investigation, K.W.; methodology, K.W. and A.W.; project administration, Y.J.; resources, L.Z.; software, A.W.; supervision, Y.J.; 
validation, A.W.; visualization, K.W.; writing—original draft, K.W.; writing—review and editing, Y.J. and P.Z. All authors have read and agreed to the published version of the manuscript.

Funding: This research was funded by Qingdao National Laboratory for Marine Science and Technology (QNLM2016ORP0402); Defense Industrial Technology Development Program (SXJQR2018WDKT02); State Key Laboratory of Structural Analysis for Industrial Equipment(S18408); and National Natural Science Foundation of China (51975032) \& (51939003).

Acknowledgments: The authors would like to thank Qingdao National Laboratory for Marine Science and Technology.

Conflicts of Interest: The authors declare no conflict of interest.

\section{Appendix A}

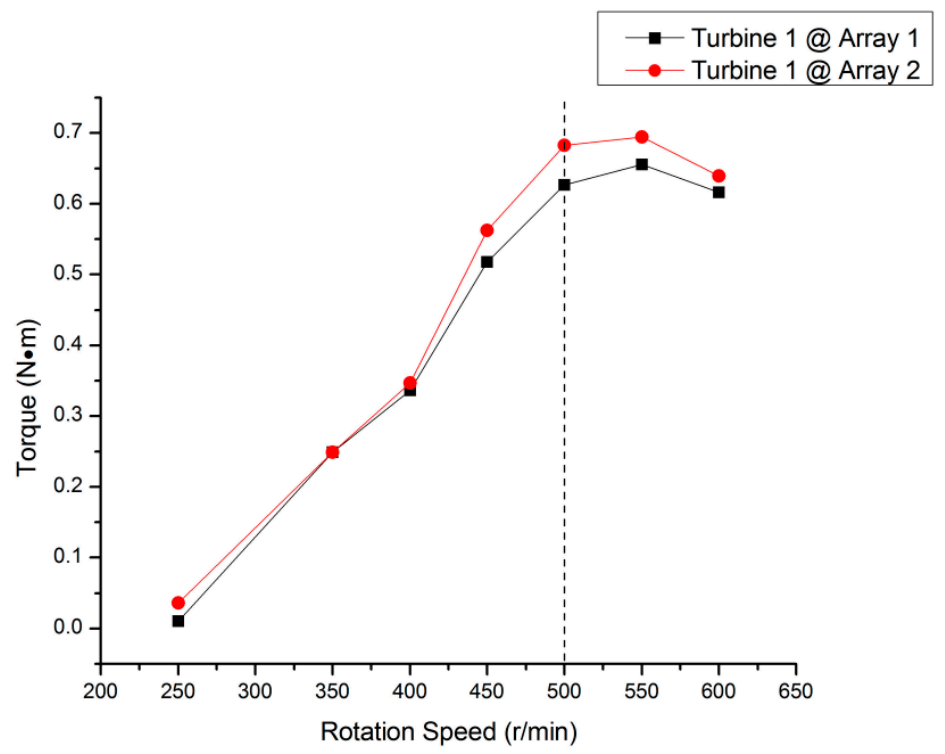

Figure A1. Torque output curves at different rotation speeds.

\section{References}

1. Balduzzi, F.; Bianchini, A.; Carnevale, E.A.; Ferrari, L.; Magnani, S. Feasibility analysis of a Darrieus vertical-axis wind turbine installation in the rooftop of a building. Appl. Energy 2012, 97, 921-929. [CrossRef]

2. Tjiu, W.; Marnoto, T.; Mat, S.; Ruslan, M.H.; Sopian, K. Darrieus vertical axis wind turbine for power generation ii: Challenges in HAWT and the opportunity of multi-megawatt darrieus VAWT development. Renew. Energy 2015, 75, 560-571. [CrossRef]

3. Rajaopalan, R.G.; Rickerl, T.L.; Klimas, P.C. Aerodynamic interference of vertical axis wind turbines. J. Propul. Power 1990, 6, 645-653. [CrossRef]

4. Wu, T.Y. Flow through a heavily loaded actuator disc. Schiffstechnik 1962, 47, 134-138.

5. Conway, J.T. Exact actuator disk solutions for non-uniform heavy loading and slipstream contraction. J. Fluid Mech. 2000, 365, 235-267. [CrossRef]

6. Madsen, H.A. The Actuator Cylinder-A Flow Model for Vertical Axis Wind Turbines. Ph.D. Thesis, Aalborg University Centre, Aalborg, Denmark, 1982.

7. Sørensen, J.N.; Shen, W.Z. Numerical modeling of wind turbine wakes. J. Fluids Eng. Trans. ASME 2002, 124, 393-399. [CrossRef]

8. Shen, W.Z.; Zhang, J.H.; Sørensen, J.N. The actuator surface model: A new navier-stokes based model for rotor computations. J. Sol. Energy Eng. Trans. ASME 2009, 131, 110021-110029. [CrossRef]

9. Medici, D.; Alfredsson, P.H. Measurements on a wind turbine wake: 3D effects and bluff body vortex shedding. Wind Energy 2006, 9, 219-236. [CrossRef]

10. Araya, D.B.; Colonius, T.; Dabiri, J.O. Transition to bluff-body dynamics in the wake of vertical-axis wind turbines. J. Fluid Mech. 2017, 813, 346-381. [CrossRef] 
11. Zanforlin, S.; Nishino, T. Fluid dynamic mechanisms of enhanced power generation by closely spaced vertical axis wind turbines. Renew. Energy 2016, 99, 1213-1226. [CrossRef]

12. Chen, W.; Chen, C.; Huang, C.; Hwang, C. Power output analysis and optimization of two straight-bladed vertical-axis wind turbines. Appl. Energy 2017, 185, 223-232. [CrossRef]

13. Lam, H.F.; Peng, H.Y. Measurements of the wake characteristics of co- and counter-rotating twin h-rotor vertical axis wind turbines. Energy 2017, 131, 13-26. [CrossRef]

14. Zdravkovich, M.M.; Pridden, D.L. Interference between two circular cylinders; Series of unexpected discontinuities. J. Ind. Aerodyn. 1977, 2, 255-270. [CrossRef]

15. Alam, M.M.; Moriya, M.; Sakamoto, H. Aerodynamic characteristics of two side-by-side circular cylinders and application of wavelet analysis on the switching phenomenon. J. Fluids Struct. 2003, 18, 325-346. [CrossRef]

16. Zhou, Y.; Zhang, H.J.; Yiu, M.W. The turbulent wake of two side-by-side circular cylinders. J. Fluid Mech. 2002, 458, 303-332. [CrossRef]

17. Xu, S.J.; Zhou, Y.; So, R.M.C. Reynolds number effects on the flow structure behind two side-by-side cylinders. Phys. Fluids 2003, 15, 1214-1219. [CrossRef]

18. Zou, L.; Wang, K.; Jiang, Y.; Wang, A.; Sun, T. Wind tunnel test on the effect of solidity on near wake instability of vertical-axis wind turbine. J. Mar. Sci. Eng. 2020, 8, 365. [CrossRef]

19. Battisti, L.; Persico, G.; Dossena, V.; Paradiso, B.; Raciti Castelli, M.; Brighenti, A.; Benini, E. Experimental benchmark data for H-shaped and troposkien VAWT architectures. Renew. Energy 2018, 125, 425-444. [CrossRef]

20. Ross, I.; Altman, A. Wind tunnel blockage corrections: Review and application to savonius vertical-axis wind turbines. J. Wind. Eng. Ind Aerod. 2011, 99, 523-538. [CrossRef]

21. Posa, A. Influence of Tip Speed Ratio on wake features of a Vertical Axis Wind Turbine. J. Wind. Eng. Ind. Aerod. 2020, 197, 104076. [CrossRef]

22. Torrence, C.; Compo, G.P. A practical guide to wavelet analysis. Bull. Am. Meteorol. Soc. 1998, 79, 61-78. [CrossRef]

23. Farge, M. Wavelet transforms and their applications to turbulence. Ann. Rev. Fluid Mech. 1992, 24, $395-457$. [CrossRef]

24. Howell, R.; Qin, N.; Edwards, J.; Durrani, N. Wind tunnel and numerical study of a small vertical axis wind turbine. Renew. Energy 2010, 35, 412-422. [CrossRef]

25. Tescione, G.; Ragni, D.; He, C.; Ferreira, C.J.S.; van Bussel, G.J.W. Near wake flow analysis of a vertical axis wind turbine by stereoscopic particle image velocimetry. Renew. Energy 2014, 70, 47-61. [CrossRef]

26. Posa, A.; Parker, C.M.; Leftwich, M.C.; Balaras, E. Wake structure of a single vertical axis wind turbine. Int. J. Heat Fluid Flow 2016, 61, 75-84. [CrossRef]

27. Sumner, D.; Heseltine, J.L.; Dansereau, O.J.P. Wake structure of a finite circular cylinder of small aspect ratio. Exp. Fluids 2004, 37, 720-730. [CrossRef]

28. Kraichnan, R.H. Inertial ranges in two-dimensional turbulence. Phys. Fluids 1967, 10, 1417-1423. [CrossRef]

29. Maassen, S.R.; Clercx, H.J.H.; van Heijst, G.J.F. Self-organization of quasi-two-dimensional turbulence in stratified fluids in square and circular containers. Phys. Fluids 2002, 14, 2150-2169. [CrossRef]

30. Schneider, K.; Farge, M. Decaying two-dimensional turbulence in a circular container. Phys. Rev. Lett. 2005, 95, 244502. [CrossRef]

31. Clercx, H.J.H.; Maassen, S.R.; van Heijst, G.J.F. Spontaneous spin-up during the decay of 2D turbulence in a square container with rigid boundaries. Phys. Rev. Lett. 1998, 80, 5129-5132. [CrossRef]

32. Molenaar, D.; Clercx, H.J.H.; Van Heijst, G.J.F. Transition to chaos in a confined two-dimensional fluid flow. Phys. Rev. Lett. 2005, 95, 104503. [CrossRef] [PubMed]

33. Frisch, U.; Sulem, P.L. Numerrical simulation of the inverse cascade in two-dimensional turbulence. Phys. Fluids 1984, 27, 1921-1923. [CrossRef]

34. Qian, J. Inverse energy cascade in two-dimensional turbulence. Phys. Fluids 1986, 29, 3608-3611. [CrossRef]

(C) 2020 by the authors. Licensee MDPI, Basel, Switzerland. This article is an open access article distributed under the terms and conditions of the Creative Commons Attribution (CC BY) license (http://creativecommons.org/licenses/by/4.0/). 